\title{
Experiencia en el Servicio de Cirugía General de un hospital privado en México durante la pandemia por SARS-CoV-2
}

\author{
Experience in the General Surgery Service of a private hospital \\ in Mexico during the SARS-CoV-2 pandemic
}

\author{
Jorge Andrés Myers Esmenjaud,* Diana Gabriela Maldonado Pintado, \\ Salvador Medina González, ${ }^{\S}$ Ricardo Daniel Romero Morelos \\ Citar como: Myers EJA, Maldonado PDG, Medina GS, Romero MRD. Experiencia en el Servicio de Cirugía General de un hospital \\ privado en México durante la pandemia por SARS-CoV-2. Acta Med. 2021; 19 (1): 81-85. https://dx.doi.org/10.35366/98575
}

\section{Resumen}

Desde que el COVID-19 fue declarado pandemia, el Servicio de Cirugía General del Hospital Ángeles Pedregal adoptó medidas específicas para el cuidado de los pacientes, del personal médico y paramédico dentro de las áreas quirúrgicas mediante una reorganización en la operatividad de las cirugías. Objetivo: Describir si las medidas implementadas en la operatividad del Servicio de Cirugía General durante la pandemia corresponden a las recomendaciones internacionales y si éstas se vieron reflejadas benéficamente en la calidad de atención intrahospitalaria. Material y métodos: Estudio descriptivo en el que se incluyeron diferentes variables y se registraron de forma consecutiva los procedimientos quirúrgicos que, dependiendo de la fase de la pandemia en México, fueron 133 para la fase uno, para la fase dos 48 y para la fase tres 60 , esto comparado de manera retrospectiva con la operatividad quirúrgica antes de la pandemia. Resultados: Disminución notable de los procedimientos quirúrgicos electivos con prioridad para la cirugía de urgencia, se realizó en mayor proporción cirugía laparoscópica, así como reducción de los días de estancia intrahospitalaria. Conclusión: Con las medidas operativas adoptadas se pudo tener una práctica segura dentro y fuera del quirófano, reduciendo notablemente el riesgo de contagio con resultados favorables en nuestro centro.

Palabras clave: Pandemia, COVID-19, cirugía electiva, cirugía de urgencia.

\section{Abstract}

Specific care took place at the Hospital Ángeles Pedregal General Surgery Area as soon as the COVID-19 was declared as a pandemic to take care of patients, medical personnel and paramedics in the surgical areas reorganizing the operational surgery steps. Objective: To describe if the implemented measures in the General Surgery operational service during the COVID-19 pandemic correspond to the international recommendations, and if these were beneficially reflected in the intra-hospital quality care. Material and methods: A descriptive and registry study was made including different variables and procedures depending of the pandemic in Mexico. 133 for phase 1, 48 for phase 2 and 60 for phase 3 , this compared retrospectively to the operation of the service before the pandemic. Results: Notable reduction of the elective priority surgical urgency procedures, in major proportion using laparoscopic methods as well as a reduction of number of days of the patients at the hospital. Conclusion: With the operative measures taken, it was possible to have a safe practice, both inside and outside of the surgical room for the patient and the intra-hospital personnel with favorable results in our care center.

Keywords: Pandemic, COVID-19, elective surgery, emergency surgery.
* Médico Residente de la Especialidad en Cirugía General del Hospital Ángeles Pedregal. Facultad Mexicana de Medicina de la Universidad La Salle. Ciudad de México, México.

₹ Médico Especialista en Cirugía General y Cirugía Bariátrica adscrita al Hospital Ángeles Pedregal. Ciudad de México, México.

§ Médico Especialista en Cirugía General y Cirugía de Colon y Recto adscrito al Hospital Ángeles Pedregal. Ciudad de México, México.

\section{- Médico Residente de Cirugía General. Facultad Mexicana de Medicina de la Universidad La Salle. Ciudad de México, México.}

Correspondencia:

Jorge Andrés Myers Esmenjaud

Correo electrónico: amyers_med@hotmail.com

Aceptado: 17-11-2020.

www.medigraphic.com/actamedica 


\section{INTRODUCCIÓN}

En diciembre de 2019 se reportó en Wuhan un brote de enfermedad respiratoria causada por el coronavirus 2 del síndrome respiratorio agudo severo (SARS-CoV-2). El 11 de marzo de 2020 la Organización Mundial de la Salud declaró pandemia a esta enfermedad denominada COVID-19 y surgieron medidas de distanciamiento social a nivel mundial. ${ }^{1}$ Es así que ante la inminente llegada de pacientes con COVID-19, nuestro hospital (Hospital Ángeles Pedregal) se dio a la importante tarea de planear y comenzar con un protocolo de actuación intrahospitalaria con base en las recomendaciones nacionales e internaciones para disminuir el riesgo de transmisión de esta enfermedad emergente. Entre las medidas que se implementaron destacan las siguientes: despachadores de solución antiséptica en áreas comunes, máximo de cinco personas en los elevadores, toma de temperatura a todos los visitantes, sanitización continua de las diferentes áreas hospitalarias, así como limitar a un solo acompañante por paciente hospitalizado.

Todas estas medidas fraguaron la operatividad del Servicio de Cirugía General de nuestro hospital dependiendo de la fase de la pandemia en México. De manera adicional, se implementaron medidas específicas para el cuidado de los pacientes (uso obligatorio de cubrebocas tipo N95, higiene de manos continua, toma de muestra PCR para SARS-CoV-2 y tomografía de tórax) y del personal dentro y fuera del quirófano (uso de mascarillas de alta filtración, gafas protectoras, careta, guantes y batas quirúrgicas impermeables) para así disminuir el riesgo de esparcimiento y contagio.

Objetivo: Describir la experiencia en el Servicio de Cirugía General de un hospital privado en cuanto a la operatividad y el volumen de cirugías electivas y de urgencia, utilizando una serie de variables para evaluar si existieron cambios durante las tres fases de la pandemia, hasta el 31 de mayo de 2020, fecha en que el hospital se convirtió en un centro libre de COVID-19. De acuerdo con estos resultados describir si la implementación de estas medidas durante la pandemia se vieron reflejadas benéficamente en la calidad de atención intrahospitalaria en comparación con la operatividad del Servicio de Cirugía General antes de la pandemia.

\section{MATERIAL Y MÉTODOS}

Estudio descriptivo en el que se registraron de forma consecutiva todos los pacientes que se sometieron a algún procedimiento quirúrgico, dependiendo de la fase de la pandemia en México; se tomaron los datos de manera prospectiva de los censos diarios del hospital. Para la fase uno se incluyó 133 , para la fase dos 48 y para la fase tres 60 , lo cual da un total de 241 procedimientos.

Las variables que se incluyeron fueron las siguientes: edad, sexo, tipo de ingreso (cirugía de urgencia, cirugía electiva), tipo de cirugía (abierta, laparoscópica o asistida por robot), tiempo quirúrgico, solicitud de PCR para SARSCoV-2 y tomografía computarizada simple de tórax como complemento prequirúrgico, días de estancia intrahospitalaria y uso de equipo de protección personal.

Adicionalmente, se recolectaron datos de manera retrospectiva con una constante de flujo de cirugías de forma habitual tres meses previos al inicio de la pandemia (preCOVID-19), en los que se identificó 410 procedimientos quirúrgicos, incluyendo las mismas variables exceptuando el PCR para SARS-CoV-2 y la tomografía computarizada (TC) simple de tórax, para así comparar si existieron diferencias en la operatividad del servicio y evaluar los resultados.

\section{RESULTADOS}

En la fase uno de la pandemia (del 28 de febrero al 23 de marzo de 2020) se realizaron 133 procedimientos quirúrgicos. La media de edad fue de 52.8 años con predominio de sexo femenino (54.1\%). El 64.7\% de los pacientes ingresaron por admisión o cirugía ambulatoria y $35.3 \%$ ingresó por el Servicio de Urgencias. El tipo de cirugía que más se realizó fue la laparoscópica, seguida de la abierta y por último la asistida por robot. Los procedimientos que más se realizaron en la fase uno fueron cirugías de hernia inguinal y colecistectomías. El promedio del tiempo quirúrgico fue de 1.69 horas y para la estancia intrahospitalaria fue de 3.6 días. En esta etapa no se realizó PCR para SARS-CoV-2 ni TC de tórax a ningún paciente.

En la fase dos de la pandemia (del 23 de marzo al 20 de abril de 2020) se realizaron 48 procedimientos quirúrgicos. La media de edad fue de 47.5 años y el sexo que predominó fue el femenino (58.3\%). Se observó una tendencia similar cercana a 50\% de ingresos por admisión/ cirugía ambulatoria y por el Servicio de Urgencias. De igual manera, se realizaron más procedimientos laparoscópicos (24), seguido de cirugía abierta (23) y por último cirugía asistida por robot (1). Los procedimientos que más se realizaron en la fase dos fueron cirugía de colon/ anorrectal y cirugía oncológica. El promedio del tiempo quirúrgico fue de 1.86 horas y para la estancia intrahospitalaria fue de 6.6 días. Se realizaron seis pruebas de PCR para SARS-CoV-2 y 10 TC de tórax como complemento en esta fase.

En la fase tres de la pandemia que tuvo lugar del 2 de abril y hasta el 31 de mayo, fecha en que el hospital se convirtió en centro libre de COVID-19, se realizaron 60 
Figura 1: Tipo de ingreso por fase de pandemia.

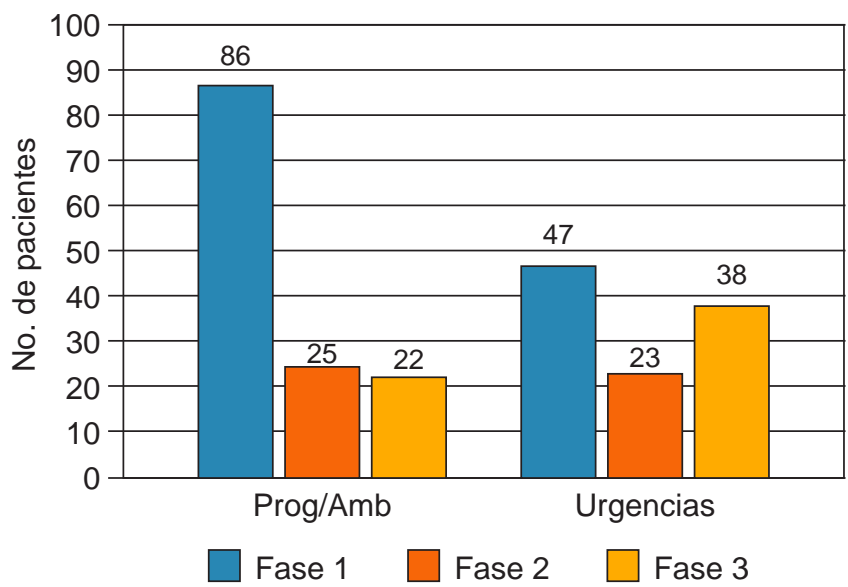

procedimientos quirúrgicos. La media de edad fue de 48.6 años, el sexo que predominó fue el femenino (58.3\%). Se observó que, a diferencia de la fase uno y dos, predominaron los ingresos por el Servicio de Urgencias sobre aquellos que fueron por admisión o cirugía ambulatoria (Figura 1). De igual manera, la cirugía que más se realizó fue laparoscópica, seguida de cirugía abierta y por último asistida por robot (Figura 2). Los procedimientos que más se realizaron en la fase tres fueron apendicetomías (15) y colecistectomías (12). El promedio del tiempo quirúrgico fue de 1.73 horas y para la estancia intrahospitalaria fue de 3.8 días. (Figura 3). En este caso se realizaron 14 pruebas de PCR SARS-CoV-2 y 30 TC de tórax como complemento en esta fase.

Por otra parte, se realizó un análisis comparativo de los datos obtenidos de los tres meses previos al inicio de la pandemia, del 01 de diciembre de 2019 al 27 de febrero de 2020 con el hospital operando de manera normal. Se observó predominio por el sexo femenino. Durante la fase previa a la pandemia por COVID-19, 56.8\% de los pacientes ingresó para cirugía programada y $43.1 \%$ ingresó por el Servicio de Urgencias, mientras que durante la pandemia los resultados fueron de 55.1 y $44.8 \%$, respectivamente (Figura 4). Con estos datos podemos observar una disminución de $42.9 \%$ en las cirugías programadas, 38.9\% en las cirugías de urgencia y $41.2 \%$ en la productividad general del servicio.

En cuanto al tipo de cirugía, predominó la técnica laparoscópica, 55.9\% en pandemia versus 53.1\% en fase preCOVID-19; seguido por la técnica abierta predominante en la fase preCOVID-19 43.5 vs $41 \%$ en pandemia y finalmente asistida por robot en 3.4 vs $3.1 \%$, respectivamente (Figura 5).

Los procedimientos que más se realizaron antes de la pandemia fueron colecistectomías, cirugía de hernia y antirreflujo, se observa un cambio gradual hacia las cirugías de urgencia durante la pandemia, específicamente en la fase tres en la que predominó la apendicectomía.

El promedio del tiempo quirúrgico fue menor antes de la pandemia (1.4 horas) en comparación a durante la pandemia (1.7 horas), esto debido a que en la fase dos hubo un aumento de las cirugías de colon y recto, así como las oncológicas. Para la estancia intrahospitalaria fueron de 3.1 días antes de la pandemia en comparación con 4.6 días durante la pandemia, esto en relación con el tipo de cirugías efectuadas en cada fase.

Durante la pandemia se identificaron en el servicio tres pacientes con COVID-19 confirmado por PCR y cuatro pacientes sugestivos por tomografía que posteriormente resultaron negativos, todos asintomáticos, fueron egresados a la brevedad y no presentaron ninguna complicación en su perioperatorio. Asimismo, sólo seis profesionales de la salud resultaron positivos para COVID-19 en nuestro servicio, fueron asintomáticos y se aislaron debidamente 14 días hasta obtener una segunda prueba negativa.

Figura 2:

Tipo de cirugía dependiendo la fase de la pandemia.

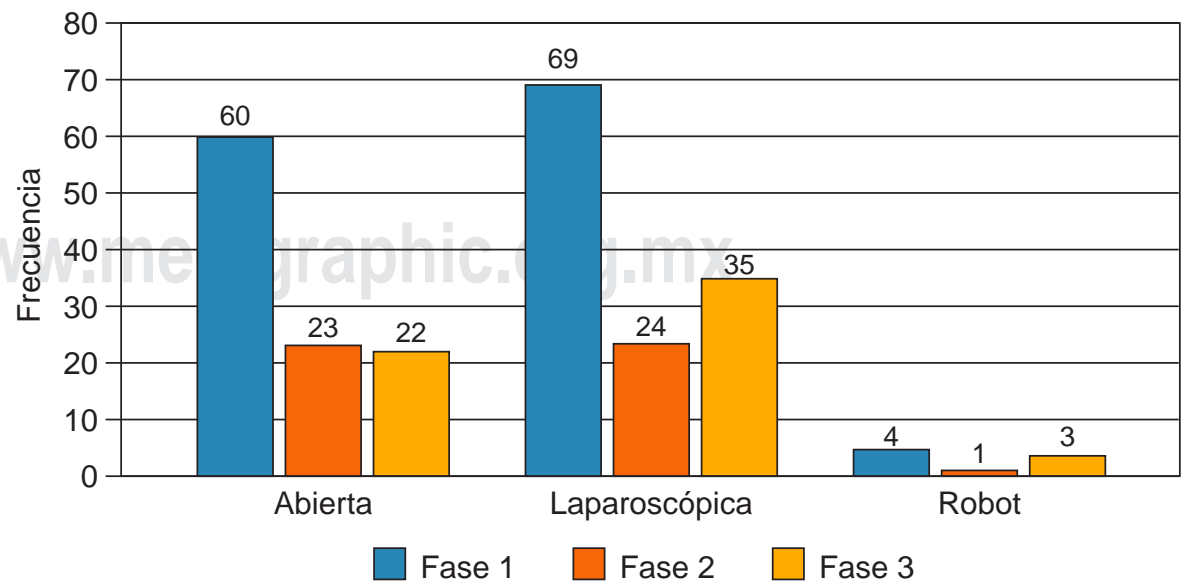


Figura 3: Promedio de días de estancia intrahospitalaria dependiendo la fase de la pandemia.

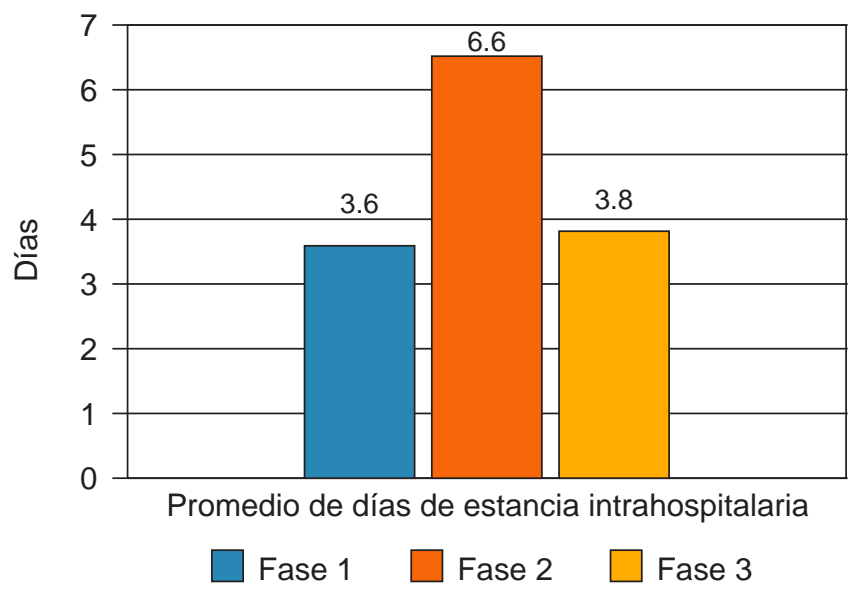

\section{DISCUSIÓN}

Desde que se declaró la pandemia por COVID-19, en México, se inició la implementación de las medidas de mitigación. Dentro de estas acciones se implementó la suspensión de actividades escolares, actividades no esenciales, restricción de la movilidad, entre otras. En los hospitales públicos y privados se realizaron consensos de las modificaciones en los planes de trabajo y logística de cada servicio. Una de las más importantes fueron los cambios en la frecuencia de procedimientos quirúrgicos programados, dando prioridad a las cirugías de urgencia.

Por tales motivos, desde que fue declarada la fase dos de la contingencia sanitaria en nuestro país, en el Servicio de Cirugía General del Hospital Ángeles Pedregal se ordenó la suspensión de reuniones y sesiones académicas y se acortaron los horarios de trabajo a jornadas no más largas de 24 horas. Estos cambios se consensuaron con el fin de disminuir el riesgo de infección del personal médico.

En una epidemia es importante definir e identificar a los grupos de riesgo de complicaciones por la infección. En el caso de SARS-CoV-2 se identificaron como poblaciones de riesgo para infecciones graves a los pacientes de edad avanzada (mayores de 65 años) y los pacientes con enfermedades crónico-degenerativas. La infección por SARS-CoV-2 demostró una alta transmisibilidad a nivel nosocomial, incluyendo al personal de salud; por lo que se considera un grupo de riesgo por su situación laboral. ${ }^{2}$

La transmisión viral puede ocurrir hasta tres días antes de que los pacientes puedan volverse sintomáticos. La eliminación viral es, por lo general, indetectable a los 21 días después de la infección. ${ }^{3}$ Es por ello por lo que diversos grupos y asociaciones afines a Cirugía General publican diferentes recomendaciones para el manejo de cirugías en temporada de COVID-19. Entre algunas destacan que cada hospital, sistema de salud y cirujano revisen cuidadosamente todos los procedimientos electivos programados con un plan para minimizar, posponer o cancelar operaciones programadas, endoscopias u otros procedimientos invasivos programados. ${ }^{4}$

Se puede definir como procedimientos invasivos electivos no esenciales a aquellos procedimientos que se programan con anticipación, ya que no involucra una emergencia médica. A medida que el brote de COVID-19 se propaga por todo el país, las autoridades se han dado a la tarea de implementar recomendaciones para la reorganización de la cirugía electiva. ${ }^{5}$

Algunos aspectos relevantes que se han documentado en los servicios de cirugía general son: reducción de la cirugía programada, reducción de las jornadas laborales y la implementación de métodos de screening preoperatorio para disminuir el riesgo de contagio, lo cual contribuyó a la caída global de la actividad quirúrgica. .,7 $^{2}$

Aunque investigaciones anteriores han demostrado que la laparoscopia puede conducir a la aerolización de virus transmitidos por la sangre, no hay evidencia que indique que este efecto se observe en COVID-19. Para los procedimientos mínimamente invasivos se debiera considerar seriamente el uso de dispositivos para filtrar el $\mathrm{CO}_{2}$ liberado para partículas en aerosol. ${ }^{8}$ Otro de los aspectos fundamentales para el equipo médico en quirófano es un adecuado uso de equipos de protección personal. Antes de realizar un procedimiento de generación de aerosol, se recomienda utilizar máscara quirúrgica adecuada, preferentemente N95, y protección ocular como gafas o caretas. ${ }^{9}$

Figura 4: Comparativa del tipo de ingreso antes y durante la pandemia.

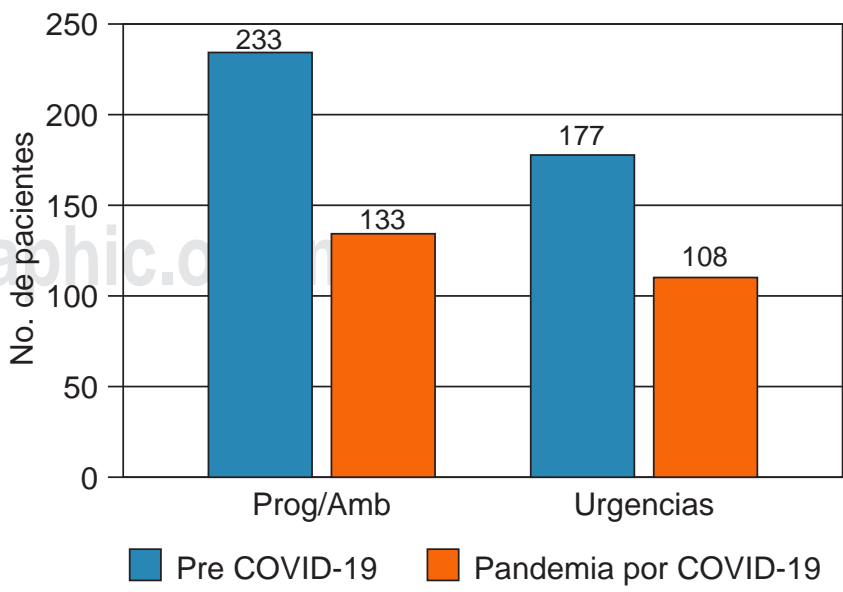


Figura 5: Comparativa de porcentaje del tipo de cirugía antes y durante la pandemia.

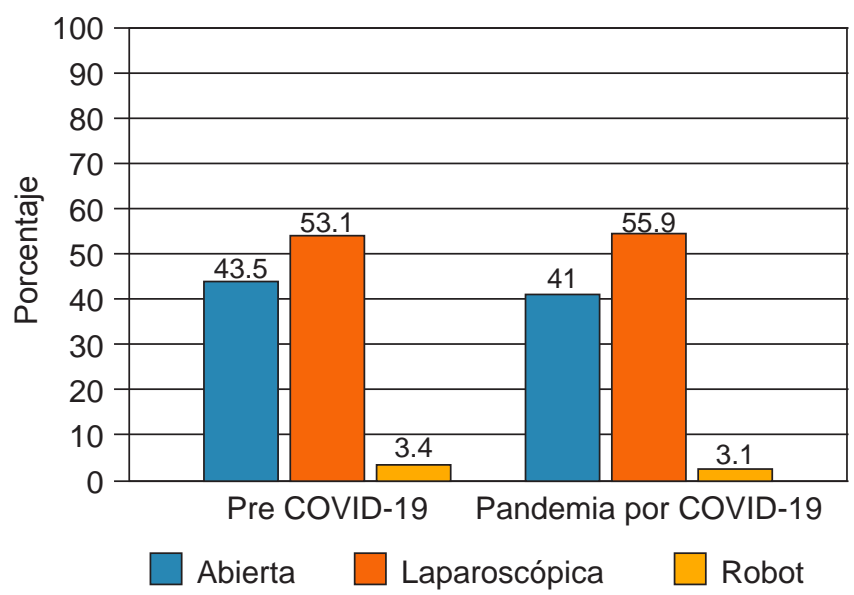

Al implementar las medidas recomendadas y al contar con los recursos necesarios se evitaron fuentes de contagios, lo cual evidencia que se siguieron las recomendaciones al observar un bajo número de pacientes y de personal infectado. En nuestro estudio se observó una disminución notable del volumen de cirugías antes y durante la pandemia donde existió inversión entre los ingresos programados o ambulatorios, más frecuentes en la fase uno y menos frecuentes en la fase tres. De igual manera, predominaron las cirugías de urgencia en la fase tres. En la fase dos se observó una igualdad entre las cirugías programadas y las cirugías de urgencia. El abordaje quirúrgico que predominó en las tres fases fue la laparoscópica. El tiempo promedio de cirugía fue proporcional a los días de estancia intrahospitalaria. Durante la fase tres se solicitaron con mayor frecuencia pruebas diagnósticas para COVID-19 y tomografía de tórax como complemento prequirúrgico, estas medidas junto con las antes descritas seguirán vigentes hasta que no exista una terapia curativa segura para esta enfermedad.

\section{CONCLUSIÓN}

Ante esta nueva enfermedad emergente, en nuestro hospital se observó una disminución de las cirugías programadas durante la fase dos y tres, siendo proporcional el tiempo quirúrgico en relación con los días de estancia intrahos- pitalaria. En su mayoría los procedimientos se realizaron por vía laparoscópica.

Se adecuaron espacios y equipos específicos destinados para pacientes con COVID-19 y aquellos que requirieron cirugía fueron protocolizados de manera adecuada para contar con una práctica segura dentro y fuera del quirófano, reduciendo notablemente el riesgo de contagio tanto para el paciente como para el personal intrahospitalario, con resultados favorables en nuestro centro.

\section{REFERENCIAS}

1. Gorbalenya AE, Baker SC, Baric RS, de Groot RJ, Drosten C, Gulyaeva AA et al. Severe acute respiratory syndrome-related coronavirus: the species and its viruses - a statement of the Coronavirus Study Group. BioRxiv. 2020. [Accessed on February 12, 2020] Available in: https://www.biorxiv.org/content/10.1101/ 2020.02.07.937862v1

2. Aragón-Nogales R, Vargas-Almanza I, Miranda-Novales MG. COVID-19 por SARS-CoV-2: la nueva emergencia de salud. Rev Mex Pediatr. 2019; 86 (6): 213-218. doi: 10.35366/91871.

3. He X, Lau EHY, Wu P, Deng X, Wang J, Hao X et al. Temporal dynamics in viral shedding and transmissibility of COVID-19. Nat Med. 2020; 26 (5): 672-675.

4. American College of Surgeons. COVID-19: guidance for triage of nonemergent surgical procedures. March 13, 2020. [Accessed June 17, 2020] Available in: https://www.facs.org/covid-19/clinical-guidance/ elective-surgery

5. Diaz A, Sarac BA, Schoenbrunner AR, Janis JE, Pawlik TM. Elective surgery in the time of COVID-19. Am J Surg. 2020; 219 (6): 900-902. doi: 10.1016/j.amjsurg.2020.04.014.

6. Álvarez Gallego M, Gortázar de Las Casas S, Pascual Migueláñez I, Rubio-Pérez I, Barragán Serrano C, Álvarez Peña E et al. SARS-CoV-2 pandemic on the activity and professionals of a General Surgery and Digestive Surgery Service in a tertiary hospital. Cir Esp. 2020; 98 (6): 320-327.

7. Cano-Valderrama O, Morales X, Ferrigni CJ, Martín-Antona E, Turrado V, García A et al. Acute Care Surgery during the COVID-19 pandemic in Spain: Changes in volume, causes and complications. A multicentre retrospective cohort study. Int J Surg. 2020; 80: 157-161. doi: 10.1016/j.ijsu.2020.07.002.

8. Recomendaciones de la Sociedad de Cirujanos Gastrointestinales y Endoscópicos Americanos y la Asociación Europea de Cirugía Endoscópica sobre la respuesta quirúrgica a la crisis de COVID-19. March 30, 2020. [Accessed June 17, 2020] Available in: https://www. sages.org/recommendations-surgical-response-covid-19

9. Centros para el Control y la Prevención de Enfermedades, Centro Nacional de Enfermedades Infecciosas Emergentes y Zoonóticas (NCEZID), División de Promoción de la Calidad de la Atención Médica (DHQP). January 7, 2016. [Accessed June 17, 2020] Available in: https://www.cdc.gov/infectioncontrol/basics/transmission-basedprecautions.html 